\title{
Zur Verbreitung der Strukturböden und Wüsten in Island ${ }^{1}$
}

\author{
Von Martin Schwarzbach, Universität Köln
}

\section{Mit 8 Abbildungen}

$\mathrm{Zusammenfassung.} \mathrm{Aus} \mathrm{Island} \mathrm{sind} \mathrm{u.a.} \mathrm{Steinringe} \mathrm{öfters} \mathrm{erwähnt} \mathrm{und} \mathrm{be-}$ schrieben worden. Sie sind meist relativ klein und nicht an Dauerfrostboden geknüptt. Über ihre Verbreitung gibt es nur unvollständige und z. T. unrichtige Angaben. In den inneren Hochebenen sind „Pflasterböden" häufig, aber Steinringe fehlen dort - wenigstens in sehr weiten Gebieten ganz. Die Ursache liegt vor allem in dem sehr wasserdurchlässigen Substrat aus vulkanischen Tuffen und Laven; dazu kommt der relativ geringe Niederschlag. Der Wassermangel im Boden läßt Bodenfrosterscheinungen und die Bildung von Strukturböden zurücktreten.

Auf die gleiche Ursache geht die große Verbreitung der W ü s t e n in Island zurück, die (ohne die Gebirge) $1 / 3$ des Landes einnehmen. Die isländischen Wüsten sind vorwiegend edaphisch und nicht so sehr klimatisch bedingt. - Es wird eine Einteilung der Wüsten auf der Erde in heiße Wüsten, Kältewüsten und edaphisch bedingte Wüsten gegeben.

$\mathrm{Summary}$. Stone-rings (and other patterned ground) found in Iceland have often been described. They are mostly relatively small and not caused by permafrost. 'Their distribution is known only incompletely. Stone-pavements are frequently found in the interior highlands, but there are no stone-rings at least in the rather extended areas which I was able to inspect. The cause is to be sought in the very permeable ground (volcanic tuffs and lavas), combined with the relatively small precipitation.

The same cause holds good for the wide distribution of dese r ts in Iceland. They occupy $1 / 3$ of the whole island (mountains not included). The Icelandic deserts result principally from edaphic - and not so much climatic - conditions. - As a conclusion the paper suggests a division of the deserts of the earth into hot deserts, cold deserts and edaphically caused deserts.

$\mathrm{R}$ é $\mathrm{s}$ u $\mathrm{m}$ é. On a décrit beaucoup de sols polygona ux (et d'autres sols périglaciaires) d'Islande. En general, ils sont rélativement petits et on ne les trouve pas sur du pergélisol (quj n'existe d'ailleurs pratiquement pas en Islande). On ne connaît que très incomplètement leur distribution. Dans les plaines intérieurs, on trouve souvent des dallages de pierres, mais pas de sols polygonaux (aux moins dans des régions très étendues). Il faut en chercher la cause surtout dans le très perméable substrat de tufs et de laves volcaniques à qui s'ajoute une précipitation restrainte.

$\mathrm{La}$ même cause est responsable de l'extension très grande des $\mathrm{d}$ é $\mathrm{s}$ e $\mathrm{r} \mathrm{ts}$ en Islande. Ils couvrent (excepté les montagnes) $1 / 3$ de toute l'île. Les déserts islandais sont dus principalement à des causes édaphiques et non pas à des causes climatiques. - Nous proposons une division des déserts de la terre en déserts chauds, déserts froids et déserts à base édaphique.

\section{N H A L T}

Einleitende Bemerkungen

Zur Erforschungs-Geschichte der isländischen Strukturböden

Zur Verbreitung der Steinringe

Pflasterböden

Zur Verbreitung der Wüsten in Island

Definition und Einteilung der Wüsten auf der Erde

Schlußbemerkung

Dank

Schriftenverzeichnis.

\section{Einleitende Bemerkungen}

In Island kann man an zahlreichen Stellen Strukturböden sehen. Im folgenden werden einige Angaben über ihre Verbreitung mitgeteilt. Ich bemerke, daß es sich um Beobachtungen handelt, die ganz nebenbei gewonnen wurden; das Thema soll also nicht etwa erschöpfend behandelt werden. Eine vollständige Karte der Strukturböden könnten wohl

1) Vortrag, gehalten auf der Hauptversammlung der Deutschen Quartärvereinigung am 23. September 1962 in Nürnberg. 
überhaupt nur isländische Kollegen geben. Trotzdem ergeben sich vielleicht auch aus meiner fragmentarischen Darstellung einige neue Gesichtspunkte für weitere Arbeiten auf diesem Gebiet.

In Island findet man von "Strukturböden" im weitesten Sinne vor allem:

1. Auf Weidegelände die Grashügel der Thuf u r. Über ihre Beziehungen zu den „Bülten“ der Alpen hat soeben S. Müller (1962) eine Studie veröffentlicht.

2. Großmaschige Spaltennetze von der Größenordnung einiger $10 \mathrm{~m}$; sie werden als Reste von Tundrenpolygonen gedeutet (vgl. z. B. Bout, Corbel etc. 1955, S. 492; Derruau 1962, pl. 19 A).

3. Stein ringe und (auf geneigtem Gelände) $S$ teinstreif en.

Nur von den Stein ringen ( $P$ Polygonalböden“) und den von ihnen abgeleiteten Steinstreifen soll im folgenden die Rede sein. Einen Eindruck von diesen Gebilden vermitteln Abb. 1-3. Sie haben meist Durchmesser von nur einigen $\mathrm{dm}$, sind also relativ klein. Sie sind nicht an Dauerfrostboden gebunden; Island hat heute höchstens ganz lokal ständig gefrorenen Boden (vgl. z. B. S. Thórarinsson 1951). Vor 50-60 Jahren war das offenbar wenigstens stellenweise etwas anders, wie sich aus Angaben von THoroddsen (1913) ergibt. Aber da die mittleren Jahrestemperaturen in Reykjavik für die Jahre $1901-1930+4.5^{\circ}$ betrugen und sie in anderen Orten nicht viel tiefer liegen, kann auch damals die Verbreitung nicht sehr groß gewesen sein.

\section{Zur Erforschungs-Geschichte der isländischen Strukturböden}

Die Erforschung der Strukturböden ist in Island - wie in andern Gebieten der Erde durch die Spitzbergen-Exkursion des Internationalen Geologenkongresses 1910 in Gang gekommen. Spethmann und Thoroddsen berichteten 1912 und 1913 wohl als Erste speziell darüber, und später hat eine ganze Reihe von Islandreisenden entsprechende Beobachtungen mitgeteilt, meist nur beiläufig, doch z. T. auch als Grundlage für grundsätzliche Ausführungen über die Genese der Steinringe (z. B. Poser 1931, Steche 1933, Rutten 1951, Bout 1953). Abbildungen von isländischen Steinringen finden sich ferner u. a. bei Hawkes 1924, Bout, Corber etc. 1955, Válek 1959, Jux 1960, Schwarzbach 1961. S. MÜLLER 1962.

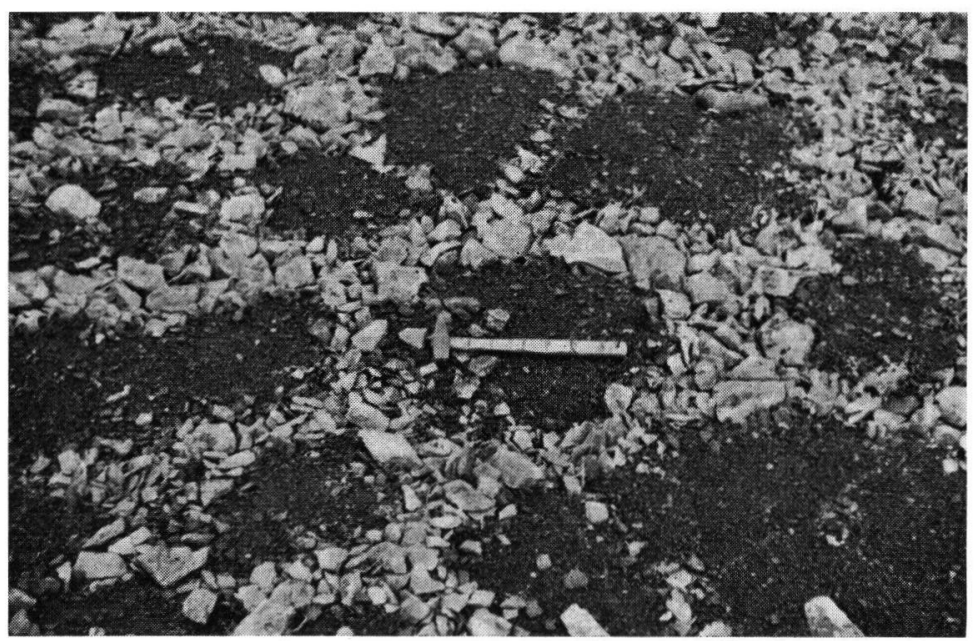

Abb. 1. Kleine Steinringe am Südhang des Kikafell bei Brjánslaekur, NW-Halbinsel, $330 \mathrm{~m}$ ü. NN. Fot. W. Friedrich, 17. 8. 1962. 


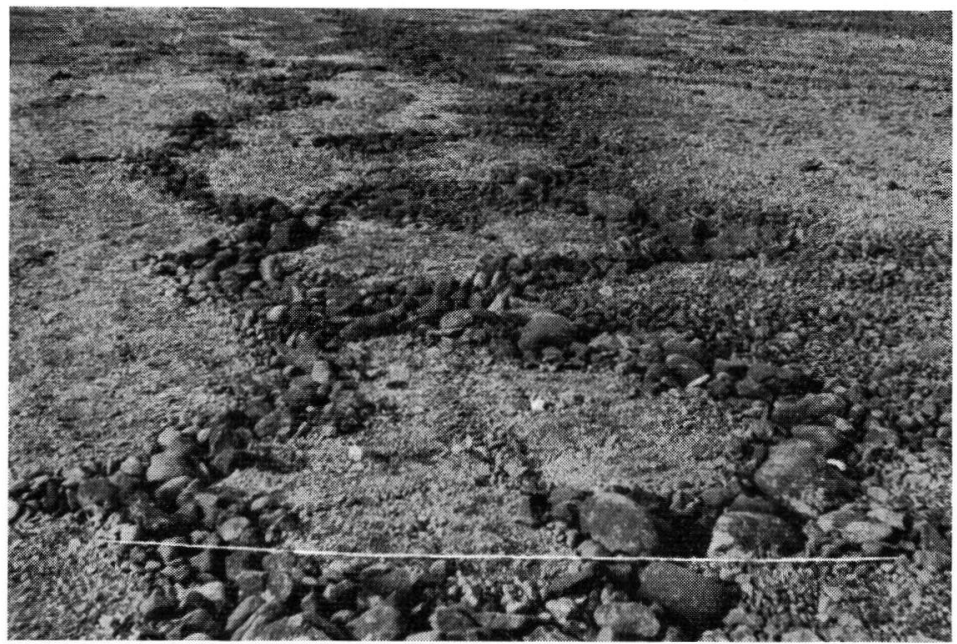

Abb. 2. Größere Steinringe am Vatnsdalvatn bei Brjánslaekur, NW-Halbinsel, ca. $10 \mathrm{~m}$ ü. NN. Der weiße Strich im Vordergrund ist ein $2 \mathrm{~m}$ langer Zollstock. Fot. W. FrIEdrich, 5. 8. 1962.

Dagegen gibt es nur recht unvollständige Angaben über die Verbreitung der Steinringe in Island. S. ThóRarinsson (1951) klassifizierte sie nach der Höhenlage (Durchmesser von $20-80 \mathrm{~cm}$ in Küstenebenen und Talauen, $60-200 \mathrm{~cm}$ auf den Inlandplateaus in $400-800 \mathrm{~m}$ Höhe, $2-10 \mathrm{~m}$ und entsprechend tiefgründig auf den Basaltplateaus in $600-1000 \mathrm{~m})$. Doch fand er bald (1953) selbst Ausnahmen und zeigte damit, daß diese Einteilung zu schematisch ist (vgl. auch Abb. 1 und 2!). C. Trous hat 1944 in seiner grundlegenden Ubersicht der Strukturböden auf der Erde versucht, auch die Verbreitung der Steinringe in Island darzustellen und zu deuten. Er konnte sich dabei nur auf wenige Literatur-Angaben stützen und verglich die kleinen Steinringe wohl richtig mit denen der

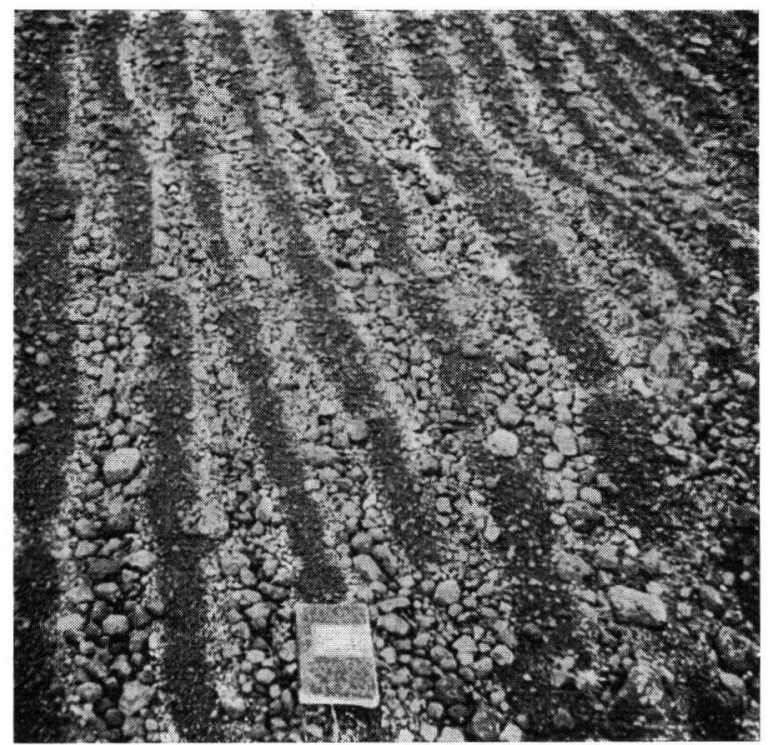

Abb. 3. Steinstreifen auf leicht geneigtem Hang über postglazialer Lava, ca. 100-150 m ü. NN. Südöstlich von Reykjavik. Maßstab: Buch. Fot. Schwarzbach, 10. 9. 1958. 
tropischen Hochgebirge; aber seine Annahme, daß in den inneren Hochländern große Ringe vorkommen, und daß diese ebenso entstanden seien wie die Dauerfrost-Steinringe Spitzbergens usw., erweist sich als nicht richtig, wie schon S. Thórarinsson (1951) klargestellt hat (vgl. auch E. M. Todtmann 1957, S. 274).

Bout, CoRbel etc. (1955) gaben sogar ein Kärtchen der Verbreitung, doch es ist lückenhaft, und die Häufung der Steinring-Signatur im SW ist wohl hauptsächlich dadurch bedingt, daß alle Islandreisenden in Reykjavik gewesen sind, also besonders viel Beobachtungen von dort mitgeteilt wurden.

Den gleichen subjektiven Akzent trägt das Nebenkärtchen ihrer Fig. 7, das die Seismizität Island darstellt (CORBEL hat die Meinung zu begründen versucht, daß e i n Faktor für die Steinsortierung die Erschütterungen durch Erdbeben seien); aus Reykjavik (wo beinahe die Hälfte der isländischen Bevölkerung wohnt!) liegen naturgemäß viele makroseismischen Beobachtungen vor, aber die instrumentellen Registrierungen aus jüngerer Zeit zeigen eine ebenso starke oder stärkere Seismizität auch an anderen (vielfach unbewohnten) Stel!en der Insel. Damit entfällt natürlich diese Erdbebenkarte Conbel's auch als Beweis für seine auch sonst unwahrscheinliche Hypothese.

Schließlich hat sich auch M. G. Rutren (1951) mit der Verbreitung der isländischen Strukturböden befaßt. Obgleich man seiner Hypothese über die Entstehung von Steinringen (=Thufur, deren Vegetation zerstört ist) wohl höchstens für ganz vereinzelte Fälle zustimmen kann, hat er doch richtig erkannt, daß in vielen "Sander-Gebieten “ Steinringe fehlen, obgleich man sie eigentlich erwarten sollte. S. THóRARInsson (1951) hat dasselbe hervorgehoben.

\section{Zur Verbreitung der Steinringe}

Auch mir fiel das, was Thórarinsson und Rutren über die Verbreitung beobachteten, schon bei meiner ersten Island-Reise 1954 auf. Noch viel mehr aber kam es mir zum Bewußtsein, als ich 1962 als Gast einer Exkursion schwedischer Quartärgeologen (mit den Kollegen S. Florin-Uppsala und C. G. WEnNer-Stockholm) eine Woche lang große Strekken des wüstenhaften Inner-Islands kennenlernte. Die Leitung der Exkursion hatte S. Thórarinsson. Die Route führte über Landmannalaugar zum westlichen Vatnajökull (Jökulheimar), am Tungnafellsjökull vorbei zum Nordrand des Vatnajökull (Dyngjajökull) und über die Askja und den Myvatn nach Akureyri. Ich habe auf dieser ganzen Fahrt kaum richtige Steinringe gesehen, obgleich ich besonders darauf achtete.

Die Ursachen dafür, daß augenscheinlich im Innern Islands über sehr weite Strecken Steinringe fehlen, können in klimatischen Verhältnissen oder in Eigenheiten des Substrats liegen. Aber das Klima ist von dem der Küstengebiete nur unwesentlich verschieden, soweit man die Temperaturen betrachtet. Wohl ist es etwas kühler, aber die Zahl der Frostwechseltage (die vor allem C. TROLL zur Erklärung der kleinen Steinringe heranzog), kann kaum grundlegend anders sein. Nach Angaben, die mir das Isländische Wetteramt freundlichst zur Verfügung stellte, hatte Reykjavik in den Jahren 1959-60 jährlich 71, Akureyri 89 Frostwechseltage. - Auch an einen Einfluß der Schneedecke wäre zu den$\mathrm{ken}^{2}$ ). Gleichfalls nach Angaben des Isländischen Wetteramtes hatte im Mittel der Jahre 1951-60 Reykjavik jährlich 65 Tage mit vollständiger Schneedecke, Sandur i Adaldal (nordöstlich von Akureyri) 111 Tage. Adaldal ist zwar nicht repräsentativ für die inneren Hochländer, aber wie die Zahlen zeigen, bestehen immerhin deutliche Unterschiede zwischen den einzelnen Orten.

Die Zusammensetzung der Böden ${ }^{3}$ ) - bisher kaum im Detail untersucht - kann schon deswegen kleine Unterschiede aufweisen, weil die Schuttdecken West- und Ost-

2) J. BüDEL wies in einer Diskussionsbemerkung zu meinem Vortrag in Nürnberg auf die Bedeutung der Schneedecke hin.

3) Unter Böden sind im folgenden (wie in der guten Übersicht, die Björn JóHanNEsson 1960 gegeben hat), alle oberflächlichen Lockerbildungen verstanden; sie zeigen in Island - im Gegensatz zu den wirklichen Böden etwa unserer Breiten - meist keine Beziehungen zu einer Vegetationsdecke. 


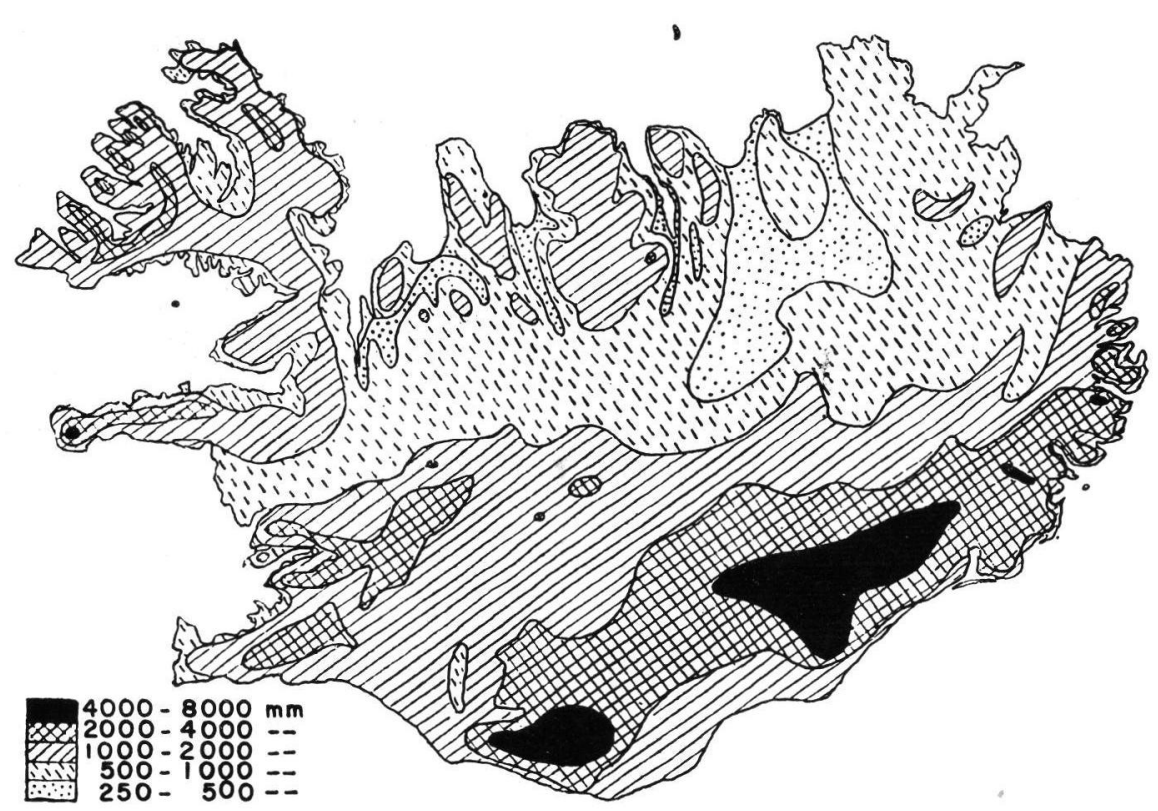

Abb. 4. Jährlicher Niederschlag in Island. Nach P. BergthóRsson aus B. JóHannesson (1960).

Islands vor allem von tertiären Basalten abzuleiten sind; sie können daher auch Komponenten einer tertiären Verwitterung enthalten, und diese hat sicherlich wesentlich mehr tonige Bestandteile geliefert als die Verwitterungsvorgänge des Eiszeitalters. Aber es gibt z. B. auch bei Reykjavik ganz junge Tuffe mit ausgezeichneten Steinringen. Es müssen also andere Faktoren hinzutreten. Ich möchte sie zum ersten darin sehen, daß in InnerIsland sehr durchlässige Böden weit verbreitet sind; junge vulkanische Tuffe und Lavadecken spielen die vorherrschende Rolle. In ihnen versickert das Wasser sehr rasch. Infolgedessen kann der Bodenfrost nur wenig wirksam werden, und der Auftauboden trocknet schnell aus. Schon ThorodDsen (1913) hat auf die Bedeutung des durchlässigen Bodens hingewiesen, ebenso E. M. Todtmann (1957).

Als zweiter Faktor kommt der geringe $\mathrm{N}$ ied e r s ch lag in Inner-Island hinzu (vgl. auch Bout, Conbel etc. 1955, S. 502 ff.). Der jährliche Niederschlag erreicht an der Südküste fast $3000 \mathrm{~mm}$ (Niederschlagsmengen bis $8000 \mathrm{~mm}$, die die Karte Abb. 4 nach P. BergthóRsson angibt, erscheinen nicht glaubwürdig). Aber nördlich vom Vatnajökull sinken die Werte auf unter $500 \mathrm{~mm}$.

Beide Faktoren wirken in derselben Richtung und müssen - vor allem wenn sie kombiniert auftreten wie in Inner-Island - für Solifluktionserscheinungen ganz andere Bedingungen schaffen als z. B. vielfach in West-Island.

Es ist bemerkenswert, daß auch in den ariden hochpolaren Gebieten (z. T. mit Jahresmitteln von -12 bis $-15^{\circ} \mathrm{C}$ und $60 \mathrm{~mm}$ Niederschlag), d. h. in den echten $\mathrm{K}$ ä $1 \mathrm{te}$ w ü s t en, Strukturböden stark zurücktreten, wie W. E. DAvies (1961) gezeigt hat.

\section{Pflasterböden}

Während man also eine polygonale Sortierung in Inner-Island immer wieder vermißt, beobachtet man in weiter Verbreitung Pf lasterböden (französisch: dallages). Mir diesem Namen bezeichnete SALOMON-CALVI (1929) ganz treffend die dichten, flachen Steinpflaster, die man z. B. aus Spitzbergen und den Alpen beschrieben hat (vgl. auch H. PHILIPP 


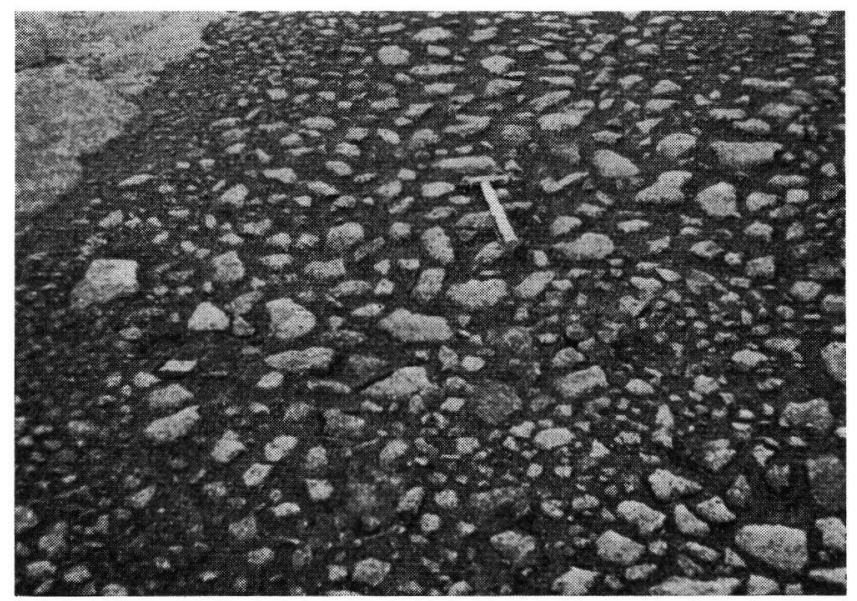

Abb. 5. Zusammenhängende Lava (Helluhraun) löst sich in ein lockeres Steinpflaster auf. Südlich Ljösufjöll (zwischen Thórisvatn und Vatnajökull). Keine polygonale Sortierung.

Fot. SchWarzbach, 21. 7. 1962

1914, Kinzl 1928, Poser 1931, Cailleux \& TAylor 1954, Bout, Córbel etc. 1955, VÁlek 1959, ferner allgemein Meinardus 1930, S. 75 ff.). Die ziemlich eckigen Steine haben meist Größen von einigen $\mathrm{cm}$, aber auch bis zu mehr als $\mathrm{dm}$ und liegen \pm dicht nebeneinander.

Schon H. Philipp (1914) hat die Ahnlichkeit mit der Hamada und den Grus- oder Kiesdecken (Serir) der heißen Wüsten betont (über diese besonders H. Montensen 1930). Dort wie in Island wird die Ausblasung von Feinmaterial aus einer Schuttdecke durch den Wind eine wichtige Rolle spielen. Auf der neuen Bodenkarte von Island sind die Pflasterböden von B. JóHANNEsson denn auch als lag-gravel bezeichnet.

Man kann gelegentlich sehen, wie sich flache Lavarücken nach den Seiten hin allmählich in einen solchen Pflasterboden auflösen (Abb. 5). Meist freilich lassen sich solche $\mathrm{Zu}-$ sammenhänge nicht erkennen (Abb. 6). Dagegen beobachtet man immer wieder unter dem

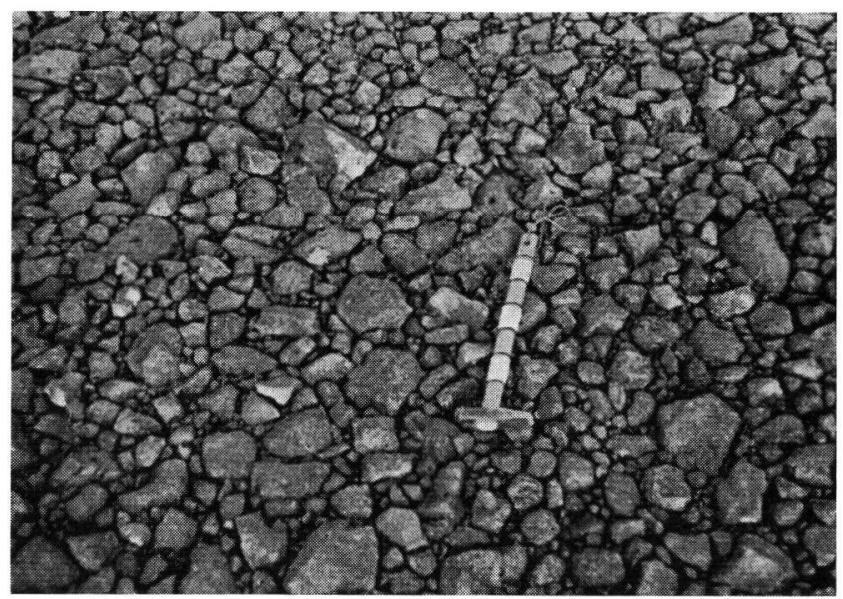

Abb. 6. Pflasterboden aus dicht gepackten Steinen. Bei Jökulheimar, vor dem westlichen Rand des Vatnajökull. Keine polygonale Sortierung. Fot. SCHWARZBACH, 21. 7. 1962 
oberflächlichen Pflaster \pm steinfreien Feinboden ${ }^{4}$ ). Wie diese ausschließlich vertikale Sortierung zustandekommt, müßte wohl noch genauer untersucht werden. Man wird zwar an das bekannte "Ausfrieren" von Steinen denken, doch bleibt es dann auffällig, daß gar keine polygonale Sortierung in der horizontalen Ebene erfolgte.

Ubrigens zeigen die heißen Wüsten auch zu diesem vertikalen Profil eine Parallele, denn „Staub unter Hamada ist sehr häufig“ (Mortensen 1930, S. 467). Hier spielen freilich solifluidale Vorgänge bestimmt keine Rolle; es handelt sich um eine „KonvergenzErscheinung".

\section{Zur Verbreitung der Wüsten in Island}

Eben wurden schon Erscheinungen in den heißen Wüsten zum Vergleich herangezogen. Auch die Verbreitung der W üsten in I s land zeigt auffallende Zusammenhänge mit dem Vorkommen oder genauer: Nicht-Vorkommen der Strukturböden.

Wüstenhafte (d.h. ganz oder fast ganz vegetationsfreie) Gebiete sind im Inneren Islands sehr weit verbreitet. Selbst wenn man die Gebirge abrechnet, die z. T. auch dazu gehören, sind immer noch ca. $30000 \mathrm{~km}^{2}$ (d. h. beinahe $30 \%$ der Insel) w ü s te $\mathrm{nh}$ a f t (Abb. 7-8). Der Eindruck, den die isländischen Wüsten auf den Reisenden machen, ist mehrfach (so von KNEBEL und $R_{E C K}$ ) eindringlich geschildert worden; schon Robert BUNSEN nannte sie 1846 „wahrhaft schauerlich“. Aber sonst hat man sie wenig beachtet und sie ihrer Entstehung nach allenfalls nebenbei als „Kältewüsten“ abgetan. Das ist eine irreführende Eingliederung.

Ich will hier nicht näher auf die ausgedehnte Diskussion über die Abgrenzung der Wüsten allgemein eingehen. Wenn man jedoch das völlige oder fast völlige Fehlen von Vegetation auch auf wenig bewegtem weiten Gelände als wesentliche Eigenschaft ansieht, so können es die Wüsten Islands mit der Sahara durchaus aufnehmen (vgl. die Definition im nächsten Abschnitt!).

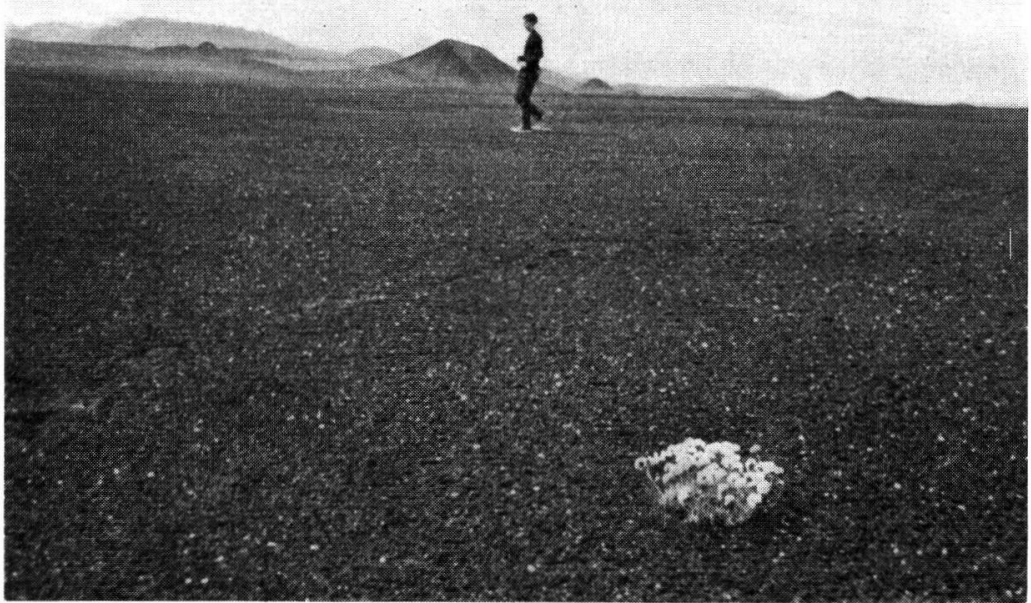

Abb.7. Landschaftsbild einer isländischen Wüste. Nördlich Vatnaöldur (zwischen Myrdals- und Hofsjökull). Die Oberfläche wird von grauschwarzem, basaltischem Sand und Kies gebildet. Ein kleiner Busch von Armeria vulgaris ist, soweit das Auge reicht, die einzige Pflanze.

Fot. Schwarzbach, 21. 7. 1962.

4) S. Thórarinsson hat mich auf der eingangs erwähnten Exkursion von 1962 mehrfach auf diese Erscheinung hingewiesen. 
In den heißen Wüsten ist es die hohe Temperatur in Verbindung mit dem sehr geringen Niederschlag, die das Fortkommen der Pflanzen verhindert; in den nicht häufigen wirklichen Kältew üsten der Polargebiete wie in Peary-Land (vgl. Fristrup 1953) oder den „Oasen“ der Antarktis (z. B. Awsjuk, Markow, Schumskij 1956, Nichols 1963) sind es die sehr tiefe Temperatur, geringer Niederschlag und die Heftigkeit der Winde. In Island jedoch ist es in erster Linie weder die Temperatur - die zwar nicht sehr hoch ist, aber ja in den Küstengebieten eine ansehnliche Vegetation ermöglicht - noch zu geringer Niederschlag; denn selbst nur $250 \mathrm{~mm}$ (meist aber über $500 \mathrm{~mm}$ ) sind bei diesen relativ kühlen Temperaturen eine ganz erhebliche Menge. Die Hauptursache liegt vielmehr im Boden, der alles Wasser verschluckt und nur besonders anspruchslosen und angepaßten (z. B. mit sehr langen Wurzeln versehenen) Pflanzen ein kümmerliches Dasein ermöglicht. Armeria vulgaris, Silene maritima, Silene acaulis u. a. sind da zu erwähnen. D i e W üs te n Is lands sind also nur beschränkt klimatisch ${ }^{5}$ ), im wesentlichen aber edaphisch bedingt. Es sind Wüsten, in denen paradoxerweise Regen und Schnee nicht viel seltener sind als bei uns.

Für diesen Typ der edaphisch bedingten Wüsten dürften die isländischen sogar die besten Beispiele auf der Erde bieten. Es ist merkwürdig, daß bisher kaum jemand darauf hingewiesen hat. Auch Erich KAISER, der 1928 den „edaphisch bedingten geologischen Vorgängen und Erscheinungen " eine besondere Untersuchung widmete, kennt einen entsprechenden Wüsten-Typ nicht. In der gut (aber etwas spitzfindig) durchdachten Landschaftsgliederung PAssarge's erscheint Island unter den Kältesteppen.

\section{Definition und Einteilung der Wüsten auf der Erde}

Wir kommen damit zu einer allgemeinen Einteilung der Wüsten auf der Erde, wie sie in der Tabelle dargestellt ist. Wir gehen dabei von folgender $\mathrm{D}$ ef in ition $\mathrm{der}$ Wüsten aus:

„Wüsten sind größere und zusammenhängende, nicht von Wasser oder Eis bedeckte Gebiete der Erdoberfläche, die ständig keine oder fast keine Vegetation tragen."

Ta belle

\section{Einteilung der Wüsten}

1. Wüsten des sommerheißen trockenen Klimas (eigentliche, vollaride Wüsten, heiße Wüsten)

Niederschlag sehr gering, Temperaturen mindestens im Sommer sehr hoch, im Winter z. T. ziemlich tief; Verdunstung sehr hoch. - Hochdruckgebiete der Subtropen und im Innern der Kontinente; ferner an tropischen und subtropischen Küsten mit kaltem Aufquellwasser.

2. Wüsten des nivalen Klimas (Kältewüsten)

Niederschlag z. T. gering, Temperaturen sehr tief (Jahrestemperatur $\left.\angle 0^{\circ}\right)$, Verdunstung $\mathrm{z}$. T. hoch. - Vor allem einzelne gletscherfreie polare Gebiete.

3. Wüsten des humiden Klimas (edaphisch bedingte Wüsten)

Niederschlag normal, Temperaturen kühl-gemäßigt; Untergrund sehr durchlässig. - Vulkanische Gebiete kühlen Klimas mit ausgedehnten Lava- und Tuffdecken6).

5) Der Einfluß des (kühlen) Klimas liegt darin, daß der Pflanzenwuchs nicht so üppig ist wie in wärmeren Breiten, und daß die chemische Verwitterung mit ihren günstigen Einflüssen auf die Bodenbildung stark zurücktritt. In diesem Zusammenhang ist beachtlich, daß der Vesu v$\mathrm{G}$ i p f e 1 - durch den Ausbruch von 1944 in einer temporäre "Wüste" verwandelt - heute schon wieder einzelne höhere Pflanzen bis hinauf zum Krater-Rand zeigt, Moose sogar im Krater (dort allerdings, soweit ich sah, an Fumarolen-Austritte gebunden; eigene Beobachtungen am 30. 9. 1962).

- Ein klassisches Beispiel für die schnelle Bewachsung im warm-feuchten Klima bietet der Krakatau.

6) Die kahlen Hänge einzelnstehender tätiger Vulkane wird man wegen ihrer beschränkten räumlichen Ausdehnung im allgemeinen nicht zu den eigentlichen "Wüsten“ rechnen. Soweit sie in feuchten wärmeren Klimaten liegen, bewachsen sie zudem relativ schnell (vgl. Fußnote 5). 


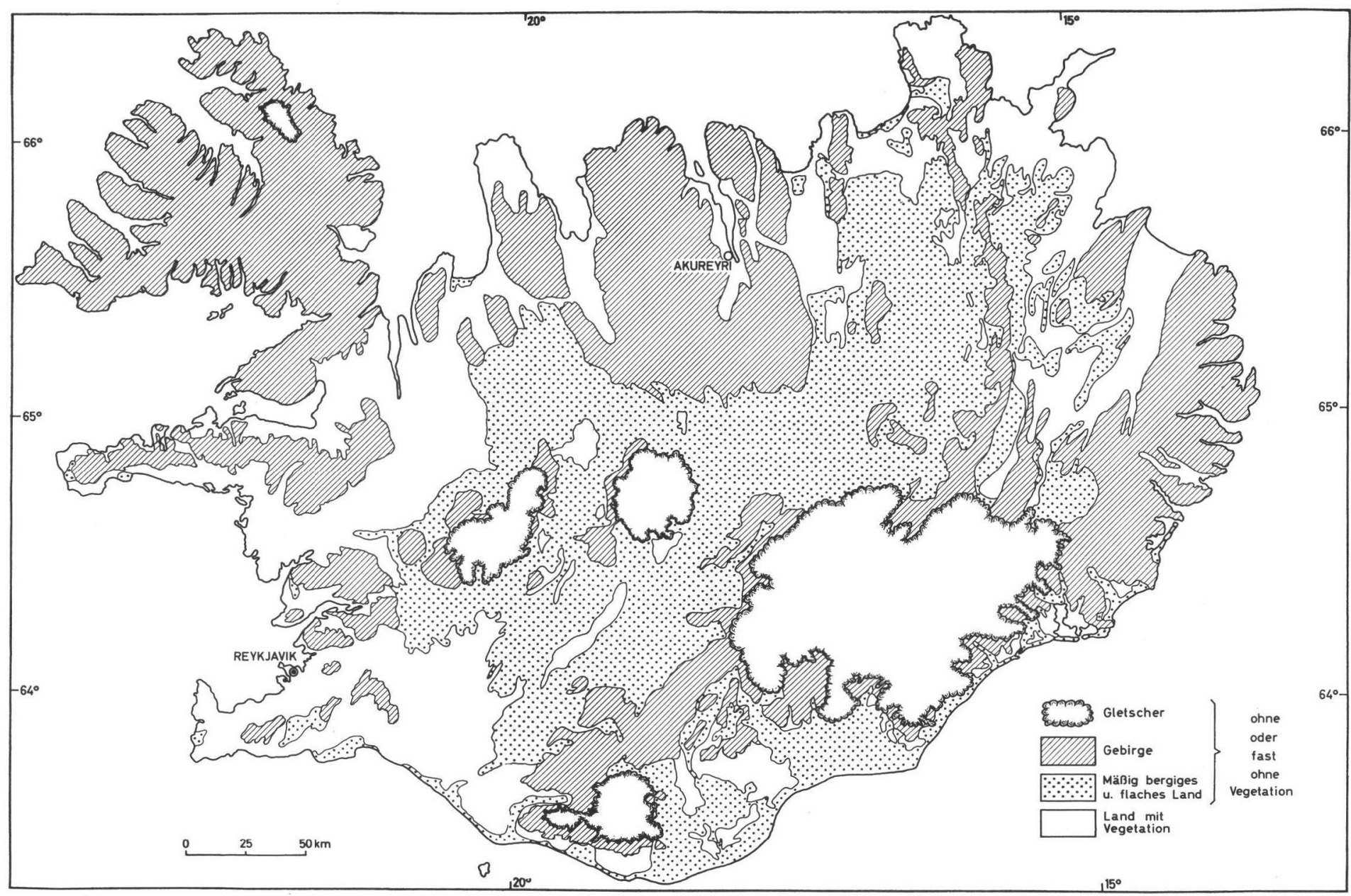

Abb. 8. Verbreitung der Wüsten in Island. Nach der Bodenkarte $1: 750000$ von B. JóHANNEsson gezeichnet. Die Wüsten nehmen fast $1 / 3$ des ganzen Landes ein. 


\section{Schlußbemerkung}

Die Verbreitung der Wüsten deckt sich in Island, wie wir sahen, mindestens in weitem Umfang mit der Verbreitung der Gebiete, in denen Strukturböden (vor allem Steinringe) fehlen. Es ist offenbar der $\mathrm{g} l \mathrm{e}$ i $\mathrm{che}$, durch die vulkanischen Gesteine besonders begünstigte Was serm a nge 1 im Boden, der sowohl den Pflanzenwuchs unmöglich macht oder erschwert, als auch für das Fehlen oder Zurücktreten der Bodenfrosterscheinungen verantwortlich gemacht werden muß. Nicht nur manche Wüsten, sondern, wenn man so will, auch die Strukturböden sind edaphisch bedingt; das Klima ist nicht der einzige maßgebende Faktor.

$\mathrm{D}$ a $\mathrm{nk}$. Ein großer Teil der Beobachtungen wurde auf Reisen gemacht, bei denen ich mich der sachkundigen Führung isländischer Fachgenossen erfreute. Besonders habe ich Jóhannes ÂsKELsson †, Prof. Trausti Einarsson und Dr. Sigurdur Thórarinsson zu danken.

\section{Schriftenverzeichnis}

Awsjuk, G. A., Markow, K. K. \& Schumskij, P. A.: [Kältewüste in der Antarktis]. - Isw. Akad. Nauk SSSR, Ser. Geogr., 16-25, Moskau 1956 (Russ.)

Bout, P.: Etudes de géomorphologie dynamique en Islande. - Expéd. polaires franç., Actual. sci. et industr., 1197, 1-219, Paris 1953.

Bout, P., Corbel, J., Derruau, M., Garavel, L. \& Péguy, Ch. P.: Géomorphologie et glaciologie en Islande centrale. - Norois, 8 (2), 463-574, Poitiers 1955.

BüdEL, J.: Die Frostschutt-Zone Südost-Spitzbergens. - Colloqu. Geogr., 6, 1-105, Bonn 1960.

Cailleux, A. \& Taylor, G.: Cryopédologie. - Expéd. polaires franç., Actual. sci. et industr., 1203, 208 S., Paris 1954.

Corbel, J.: Sols polygonaux et "terrasses marines" du Spitzberg. - Rev. Géogr. Lyon, 29, 1-28, Lyon 1954.

Davies, W. E.: Surface features of permafrost in arid areas. - Folia Geogr. Danica, 9, 48-56, Kobenhavn 1961, und in: G. O. RaAsch, Geology of the Arctic, II, 981-987, Toronto 1961.

Derruau, M.: Précis de géomorphologie. 3. Aufl. - 413 S., Paris 1962.

FristruP, B.: High Arctic deserts. - Congr. Géol. Intern. 19, Alger, VII, 91-99, Alger 1953.

Jóhannesson, B.: The soils of Iceland. - Univ. Research Inst., Dept. Agricult. Rep., B, 13, 1-140, Reykjavik 1960.

Jux, U.: Zur Geologie des Vopnafjord-Gebietes in Nordost-Island. - Geologie, Beih., 28, 1-57, Berlin 1960.

HAwKEs, L.: Frost action in superficial deposits, Iceland. - Geol. Mag., 56, 509-513, London 1924.

KaISER, E.: Uber edaphisch bedingte geologische Vorgänge und Erscheinungen. - Sitz.Ber. Bayr. Akad. Wiss., Math.-nat. Abt., 37-70, München 1928.

KinzL, H.: Beobachtungen über Strukturböden in den Ostalpen. - Peterm. Geogr. Mitt., 74, 261-265, Gotha 1928.

KNEBEL, W. v. \& ReCK, H.: Island. - 290 S., Stuttgart 1912.

Meinardus, W.: Arktische Böden. - Hdb. Bodenlehre (ed. E. Blanck), III, 27-96, Berlin 1930.

Mortensen, H.: Die Wüstenböden. - Hdb. Bodenlehre (ed. E. BlancK), III, 437-490, Berlin 1930.

MülLER, S.: Isländische Thufur und alpine Buckelwiesen - ein genetischer Vergleich. - Nat. u. Mus., 92, 267-274, 299-304, Frankfurt 1962. - - Der Boden - unsere Lebensgrundlage. 11. Der Bodenfrost. - Kosmos, 58, 542-545, Stuttgart 1962.

Nichols, R.: Geological features demonstrating aridity of Mc Murdo Sound area, Antarctica. Vortrag: Paleoclimates Conference, Newcastle, Januar 1963 (auch: Am. J. Sci., 261, 20-31, New Haven 1963).

Nielsen, N.: Contributions to physiography of Iceland. - Danske Vid. Selsk. Skrift., Nat. Mat. Afd., 9, IV, 5, 183-297, Kobenhavn 1933.

Passarge, S.: Vergleichende Landschaftskunde. 2. Kältewüsten und Kältesteppen. - 163 S., Berlin 1921.

PhILIPP, H.: Ergebnisse der W. Filchnerschen Vorexpedition nach Spitzbergen 1910. Geologische Beobachtungen. - Peterm. Mitt., Erg.-H., 179, 13-46, Gotha 1914.

Poser, H.: Beiträge zur Kenntnis der arktischen Bodenformen. - Geol. Rdsch., 22, 200-231, Stuttgart 1931.

Rutten, M. G.: Polygon soils in Iceland. - Geol. en Mijnb., 13, 161-167, s'Gravenhage 1951.

Salomon-Calvi, W.: Arktische Bodenformen in den Alpen. - Sitz.Ber. Heidelberger Akad. Wiss., Math.-nat. Kl., 1-31, Berlin-Leipzig 1929. 
SAmuelsson, C.: Studien über die Wirkungen des Windes in den kalten und gemäßigten Erdteilen. Bull. Geol. Inst. Upsala, 20, 55-230, Uppsala 1926.

Schenk, E.: Die Mechanik der periglazialen Strukturböden. - Abh. hess. Landesamt f. Bodenf., 13, 1-92, Wiesbaden 1955.

Schwarzbach, M.: Das Klima der Vorzeit. 2. Aufl. - 275 S., Stuttgart 1961.

Spethmann, H.: Über Bodenbewegungen auf Island. - Z. Ges. Erdk., 246-248, Berlin 1912.

STECHE, H.: Beiträge zur Frage der Strukturböden. - Ber. Verh. Akad. Wiss., Math.-phys. Kl., 85, 193-272, Leipzig 1933.

T'hórarinsson, S.: Notes on patterned ground in Iceland. - Geogr. Ann., 33, 144-156, Stockholm 1951. - - Islande. - Union géogr. internat., Comm. morphol. périglac., Rapports prélim. pour la 8e Assembl. génér., Washington 1952, 1-2, New York 1952. - - Anchored stone polygons at low levels within the Iceland basalt regions. - Jökull, 3, 37-38, Reykjavik 1953.

Thorodosen, Th.: Polygonböden und "thufur" auf Island. - Peterm. geogr. Mitt., 59, 253-255, Gotha 1913.

Todtmann, E. M.: Kringilsárrani, das Vorfeld des Brúarjökull am Nordrand des Vatnajökull. 1955. - N. Jb. Geol. Paläont., Abh. 104, 255-278, Stuttgart 1957.

Troll, C.: Strukturböden, Solifluktion und Frostklimate der Erde. - Geol. Rdsch., 34, 545-694, Stuttgart 1944.

VÁLEK, B.: Die Böden des Süd-Kaldidalur-Gebiets auf Südwest-Island und ihre Vegetationsbeziehungen. - Rozpr. Českosl. akad. věd, 69, 8, 1-36, Praha 1959.

Woldstedt, P.: Vergleichende Untersuchungen an isländischen Gletschern. - Jb. preuß. geol. L. A., 59 (1938), 249-271, Berlin 1939.

Manuskr. eingeg. 1. 10. 1962.

Anschrift des Verfassers: Prof. Dr. Martin Schwarzbach, 5 Köln, Zülpicher Straße 47, Geol. Institut der Universität. 